\title{
Inhibition of Autophagy Attenuated Cell Damage after OGD/R in SH-SY5Y Cells by Down-Regulating AMPK-Mediated Autophagy Signaling Pathway
}

\author{
Yanxue Zhang ${ }^{1,2}$, Hongyun $\mathrm{Li}^{*}$ \\ ${ }^{1}$ Department of Neurology, Affiliated Hospital of Qingdao University, Qingdao, China \\ ${ }^{2}$ Institute of Integrative Medicine, Qingdao University Medical College, Qingdao, China \\ Email: ${ }^{\star} 18661808653 @ 163 . c o m$
}

How to cite this paper: Zhang, Y.X. and Li, H.Y. (2019) Inhibition of Autophagy Attenuated Cell Damage after OGD/R in SH-SY5Y Cells by Down-Regulating AMPKMediated Autophagy Signaling Pathway. Open Access Library Journal, 6: e5240. https://doi.org/10.4236/oalib.1105240

Received: February 11, 2019

Accepted: February 22, 2019

Published: February 25, 2019

Copyright $\odot 2019$ by author(s) and Open Access Library Inc.

This work is licensed under the Creative Commons Attribution International License (CC BY 4.0).

http://creativecommons.org/licenses/by/4.0/

\begin{abstract}
Background: Activation of autophagy becomes a new therapeutic target for the treatment of stroke. The aim of this study was to observe the role of autophagy in the model of glucose-oxygen deprivation reoxygenation (OGD/R) in SH-SY5Y cells by interfering with AMPK-mediated autophagy signaling pathway. Methods: The effects of autophagy on OGD/R injury in SH-SY5Y cells were investigated by evaluating cell viability and morphologic change using Cell Counting Kit-8 (CCK-8) and inverted microscop, respectively. To investigate whether autophagy played a role via the AMPK-mTOR signaling pathway, the levels of phospho-AMPK, phospho-mTOR, ULK1, microtubule-associated protein 1 light chain 3 (LC3) and Beclin 1 were detected using Western blot. In order to detect changes in autophagic flow after OGD/R, autophagic flux (number of autophagosomes and autophagosomes) was detected by tandem stably expressed a tandem GFP-mRFP-LC3 construct. Results: Autophagy was low in the control group. After OGD/R, the expression of autophagy-related proteins LC3 and Beclin 1 and autophagic flux increased, phospho-AMPK and ULK1 expression increased and phospho-mTOR expression decreased, meanwhile abnormal cell morphological changes increased significantly and cell viability decreased. Inhibition of AMPK activity, the levels of phospho-mTOR, ULK1, LC3, Beclin1 and the expression of autophagic flux were opposite to those of the model group; cell abnormal morphology and structure improved, and cell viability increased. Conclusion: Inhibition of autophagy can attenuate cell damage after OGD/R in SH-SY5Y cells by down-regulating AMPK-mediated autophagy signaling pathway.
\end{abstract}




\section{Subject Areas}

Internal Medicine, Neurology

\section{Keywords}

Autophagy, OGD/R, AMPK, SH-SY5Y Cells

\section{Introduction}

Stroke is one of the leading causes of human disability and death [1]. After cerebral ischemia, short-term intravenous thrombolysis is the only treatment approved by the Food and Drug Administration for the treatment of cerebral ischemia [2]. However, thrombolysis has a 3-hour time window limit and can cause inevitable damage in perfusion [3]. Therefore, finding new therapeutic targets to protect brain tissue from reperfusion injury is critical.

The survival of nerve cells depends on the balance between cellular protein metabolism and damaged organelles [4]. This balance is mainly maintained by the two mechanisms of ubiquitin protease system and autophagy. The ubiquitin protease system mainly degrades short-lived proteins, while autophagy is the main pathway for degradation of long-lived proteins and damaged organelles [5]. Recently, autophagy can be activated by cerebral ischemia [6]. However, the role of autophagy in cerebral ischemia has been controversial. Autophagy helps neurons overcome temporary energy crises caused by cerebral infarction [7]. 3-methyladenine (autophagy inhibitor) obviously inhibited neuronal autophagy and reduced the infarct volume in rat middle cerebral artery occlusion (MCAO) model. Studies had also shown that inhibition of autophagy could reduce nerve damage in ischemic stroke [8]. However, studies had also shown that inhibition of autophagy could reduce nerve damage in ischemic stroke [9] [10]. Therefore, autophagy may be a new potential therapeutic target for the treatment of ischemic stroke.

Adenosine monophosphate-activated protein kinase (AMPK) is a cellular energy sensor that changes the ratio of intracellular AMP/ATP. AMPK is activated by phosphorylation after cerebral ischemia in rats [11]. Activated AMPK stabilizes intracellular energy balance by regulating breakdown of anabolism and autophagy [12]. Mammalian target of rapamycin (mTOR) is an atypical serine/threonine kinase belonging to the phosphoinositide 3-kinase family, a major regulator of cellular metabolism and negative feedback regulation of autophagy. Previous studies have demonstrated that AMPK can directly phosphorylate TSC1/2 complex or directly affect the Raptor subunit of mTORC1, inhibit mTORC1 activity, and induce autophagy [13] [14]. Cumulative evidence suggests that in rats with a permanent middle cerebral artery occlusion model, melatonin can activate mTOR and reduce cerebral ischemic injury [15], while rapamycin (mTOR inhibitor) aggravates brain damage [11]. The above results in- 
dicate that activation of mTOR will have neuroprotective effects. Therefore, inhibiting autophagy by activating mTOR, we have provided new ideas for the treatment of cerebral ischemia-reperfusion injury. Thus, we hypothesize that activation of mTOR by inhibiting AMPK (upstream target of mTOR) has protective effects on cerebral ischemia-reperfusion injury. OGD is widely used as an in vitro model for cerebral ischemia, showing similarities with the in vivo models of brain ischemia [16]. In the present study, we perform serial experiments by establishing an OGD/R model in SH-SY5 cells to demonstrate that inhibition of autophagy protects neurons from damage caused by OGD/R by inhibiting AMPKmediated autophagy signaling pathways.

\section{Materials and Methods}

\subsection{Cell Lines}

SH-SY5Y cell lines were purchased from Chinese Academy of Sciences Cell Bank. Cells were planted Dulbecco's modified Eagle's medium (DMEM) high glucose supplemented with $10 \%$ fetal bovine serum (FBS), placed in a $37^{\circ} \mathrm{C}, 5 \%$ $\mathrm{CO}_{2}$ incubator.

\subsection{Reagents}

Rapamycin (\#S1039, Selleck, USA); Compound C (HY-13418, MCE, USA); Cell counting kit-8 (HY-K0301, MCE, USA); DMEM/High glucose medium (SH30243.01, Hyclone, USA); Phosphate Buffered Saline (PBS) (Hyclone, USA); Trypsin (sm-2003-c, Milipore, Germany); DMEM no glucose medium (\#11966-025, GIBCO, USA); anti-phosph-AMPK Ab (\#2535, Cell Signaling Technologys, USA); anti-phosph-mTOR Ab (\#5536, Cell Signaling Technologys, USA); anti-phospho-ULK1 Ab (\#14202, Cell Signaling Technologys, USA); anti-LC3A/B Ab (\#4108, Cell Signaling Technologys, USA) and anti-Beclin 1 Ab (\#3495, Cell Signaling Technologys, USA); Enhanced BCA assay kit (No. P0010, Beyotime Institute of Biotech, China), 4,6-diamidino-2-phenylindole (DAPI, C1005, Beyotime Institute of Biotech, China); mRFP-GFP-LC3 double-labeled adenovirus (HH20180323GZH-AP01, Hanbio Biotechnology, China).

\subsection{Cell Model and Treatment}

SH-SY5Y cells were plated in 6 or 96 well for $24 \mathrm{~h}$ and established an OGD/R model when cells reached $80 \%$. DMEM high glucose medium containing $10 \%$ FBS was replaced with glucose-free serum-free DMEM medium, placed in a constant temperature $37^{\circ} \mathrm{C}$ incubator, continuously filled with oxygen-free gas $\left(90 \% \mathrm{~N}_{2}, 9 \% \mathrm{CO}_{2}\right.$ and $\left.1 \% \mathrm{O}_{2}\right)$, after $9 \mathrm{~h}$, replaced with DMEM high-sugar medium containing $10 \% \mathrm{FBS}$, and placed in an incubator at $37^{\circ} \mathrm{C}, 5 \% \mathrm{CO}_{2}$ for $4 \mathrm{~h}$.

Cells were divided into four groups after culture $24 \mathrm{~h}$, as follows: control group, model group, Compound C group, and rapamycin group. DMEM high glucose medium containing 10\% FBS was used to establish in contronl group. OGD/R model was established in the other three groups. Treatment of inhibitor 
cells with Compound C 2 hours before model construction. At the beginning of reoxygenation, normal medium was added to the model group, and the medium containing Compound C ( $5 \mu \mathrm{mol} / \mathrm{l}$ and rapamycin $(10 \mu \mathrm{mol} / \mathrm{l})$ were added to the inhibitor group and rapamycin group respectively. The cells were reoxygenated in the incubator for $4 \mathrm{~h}$.

\subsection{Cell Morphology}

In order to observe the changes in the growth status, quantity and morphology of each group of cells, five non-overlapping views were randomly selected under inverted microscope $\times 100$ after OGD/R.

\subsection{Cell Counting Kit-8}

SH-SY5Y cell were plated in 96 wells at $1 \times 10^{4}$ per well for $24 \mathrm{~h}$ and established a OGD/R model allowed when cells reached $80 \%$. After the modeling, added 90 $\mu \mathrm{L}$ of normal medium and $10 \mu \mathrm{L}$ of CCK-8 solution to each well, in $37^{\circ} \mathrm{C}$ incubator incubation $2 \mathrm{~h}$, at the same time set a blank well as a control. The $450 \mathrm{~nm}$ $\mathrm{OD}$ value of each well was measured by microplate reader.

\subsection{Western Blot Analysis}

The OGD/R model was established and they were gently rinsed with cold PBS. The cells were dissolved in buffer containing radioimmunoprecipitation assay buffer (RIPA) with both phosphatase and protease inhibitors. The cell homogenate from was collected and lysed on ice, the cell homogenate was centrifuged for $15 \mathrm{~min}$ at $4^{\circ} \mathrm{C}, 12,000 \mathrm{r} / \mathrm{min}$ in a refrigerated centrifuge (Eppendorf 5801 type, Germany) to remove cellular debris. The protein concentration in the supernatant is detected by the BCA quantitation kit. Total protein was speparated on SDS-polyacrylamide gel (Bio-Rad), transferred to a polyvinylidene difluoride (PVDF) membrane (Millipore, USA). The PVDF membranes were blocked with $5 \%$ BSA in TBST, and incubated with the following antibodies $4^{\circ} \mathrm{C}$ overnight: rabbit anti-LC3A/B (1:1000); abbit anti-phospho AMPK (1:1000), abbit anti-total AMPK (1:1000); rabbit anti-phospho mTOR (1:1000), rabbit anti-total mTOR (1:1000). After washing 3 times with the TBST, and then incubated with the HRP-labeled secondary antibodies (1:5000) for $1 \mathrm{~h}$ at room temperature. Bands were visualized with ECL method, and analyzed using Image J system. The density of each band was compared with GAPDH (1:5000) or $\beta$-actin (1:5000).

\subsection{Virus Transfection}

SH-SY5Y cells were plated in 24 wells containing coverslips at $1 \times 10^{5}$ per well and allowed to reach $50 \%-70 \%$ confluence at the time of transfection. Cells were transfected with $\mathrm{mRFP}-\mathrm{GFP}-\mathrm{LC} 3$ adenoviral vectors containing polybrene $(8 \mu \mathrm{g} / \mathrm{mL})$. Eight hours later, cells were switched to $10 \%$ serum-containing medium for $48 \mathrm{~h}$, and used for experiments. Images were captured by confocal microscope. Five non-overlapping fields were randomly selected. 


\subsection{Statistical Analysis}

The SPSS17.0 software was used for statistical analysis. Measurement data is expressed by mean \pm standard deviation (SD). One-way Anova was used to compare multiple groups. One-way ANOVA and Bonferroni post-special tests were used to determine statistical differences due to normal distribution and equal variance. It is considered that the difference of $P<0.05$ is statistically significant.

\section{Results}

\subsection{Autophagy Was Activated in SH-SY5Y Cells after OGD/R}

LC3 is an important surface substance for detecting autophagy, whlie Beclin 1 is essential for the nucleation stage of autophagy [17]. To examine autophagic activity after OGD/R, we analyzed the levels of LC3 and Beclin 1 by Western blot. As shown in Figures 1(a)-(d), the expression of LC3 and Beclin 1 were low in control group $(0.05 \pm 0.02)$, while the protein levels of LC3 and Beclin 1 increased in model group $(1.14 \pm 0.04,0.44 \pm 0.06)(t=42.90, t=8.59, P<0.01, P$ $<0.05)$ and rapa group $(1.68 \pm 0.05,0.63 \pm 0.03)(t=54.00, t=14.92, P<0.01, P$ $<0.01)$. Pretreatment with Compound C reduced the LC3 $(0.79 \pm 0.03)$ and Beclin 1 levels $(0.26 \pm 0.03)$ compared with the model group $(t=9.628, P<0.05),(t$ $=6.61, P<0.05)$.

\subsection{Autophagy Flux Was Up-Regulated in SH-SY5Y Cell in SH-SY5Y Cells after OGD/R}

To monitor autophagic flux, we established SH-SY5Y cells that stably expressed a tandem mRFP-GFP-LC3 construct. mRFP-GFP-LC3 adenovirus monitors the dynamic process of autophagosome (Yellow fluorescence) to autolysosome (red fluorescence) formation. As shown in Figure 2, we could barely see fluorescent
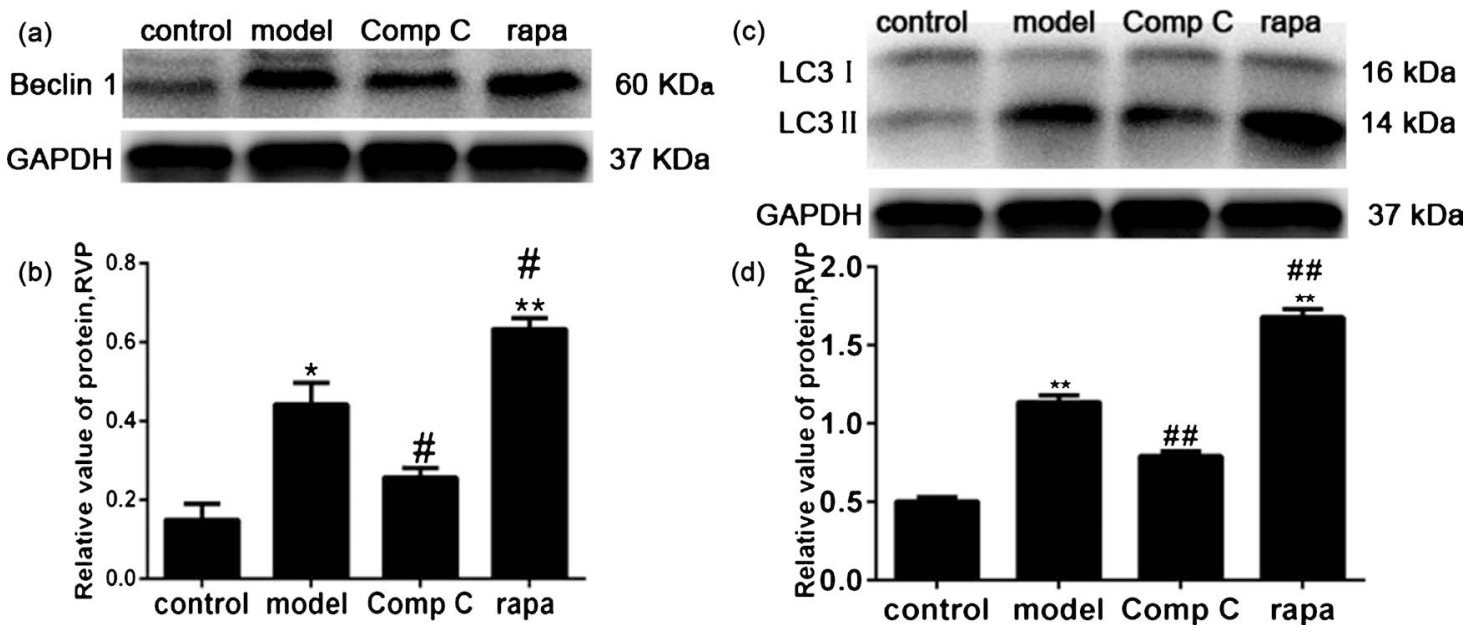

Figure 1. Autophagy is activated in SH-SY5Y cells after OGD/R. (a), (b): Representative immunoblots and quantification for analysis Beclin 1 in SH-SY5Y cells after OGD/R. (c), (d): Representative immunoblots and quantification for analysis of LC3 in SH-SY5Y cells after OGD/R. Data are represented as mean \pm SEM from two independent experiments, ${ }^{\star} P<0.05,{ }^{*} P<0.01$ vs. control group; ${ }^{\sharp} P<0.05,{ }^{\# \#} P<0.01$ vs. model group. Comp C: Compound C; rapa: rapamycin. 

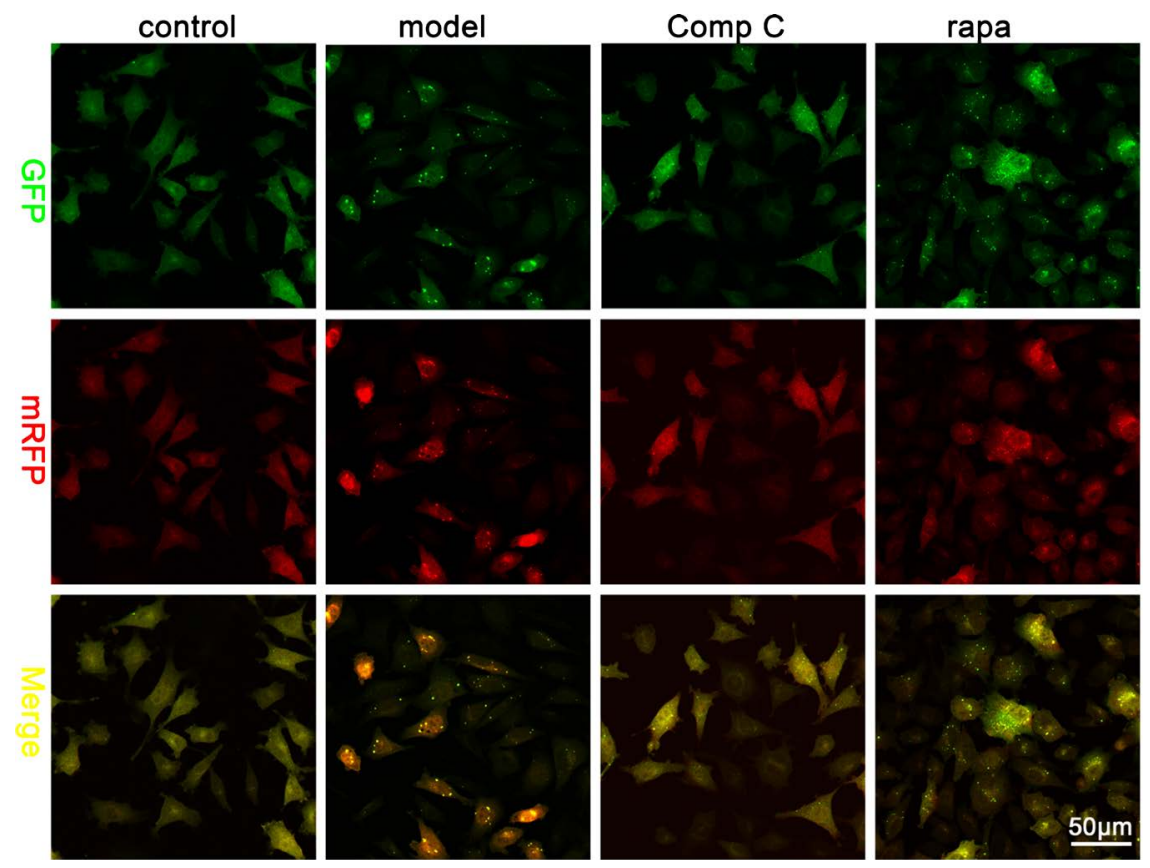

Yellow dots represent autophagosomes and red dots represent autophagic lysosomes. Scale bars: 50 $\mu \mathrm{m}$.

Figure 2. Autophagy flux was up-regulated in SH-SY5Y cell in SH-SY5Y cells after $\mathrm{OGD} / \mathrm{R}$.

dots in control group, while there were a relatively large number of fluorescent dots in model group and rapa group, compare with model group, suggesting that OGD/R and rapamycin introduced autophagy in SH-SY5Y cells. However, the inhibition of AMPK activity by Compound $\mathrm{C}$ reduces the number of fluorescent dots, intimating that autophagic flux was blocked. The above results indicated that autophagy was activated after OGD/R.

\subsection{The AMPK-mTOR Signaling Pathway Was Involved in the Process of OGD/R-Induced Autophagy}

To further obseverd the AMPK-mTOR signaling pathway after OGD/R, we first examined the expression of total-mTOR and phospho-mTOR levels by Western Blot. As shown in Figure 3(c) and Figure 3(d), there was no significant difference in the expression of total-mTOR levels among the groups $(F=3.19, P>$ $0.05)$; the phospho-mTOR level was higher in the control group $(0.68 \pm 0.05)$, while the OGD/R $(0.24 \pm 0.05)$ and rapamycin $(0.17 \pm 0.03)$ were decrease $(t=$ 24.87, $P<0.01),(t=20.24, \mathrm{P}<0.01)$. The effect of $\mathrm{OGD} / \mathrm{R}$ and rapamycin was blocked by pretreatment with Compound C $(0.37 \pm 0.04),(t=14.82, P<0.01)$. As shown in Figure 3(a) and Figure 3(b), total-AMPK and phospho-AMPK (Thr172) were detected by Western Blot. No change in total-AMPK protein expression as observed in each group $(F=1.01, P>0.05)$. AMPK was phosphorylated and activated at the Thr172 site by $\mathrm{OGD} / \mathrm{R}$ and rapamycin. However, treatment with Compound C will prevent the expression of AMPK. Compare with the control group, the ratio of phospho-AMPK/total-AMPK increased in 
model group (0.4 \pm 0.07$),(t=9.29, P<0.05)$. Pretreatment with Compound C $(0.24 \pm 0.04)$ reduced the ratio, compare to the model group $(t=15.40, P<0.01)$. However, treatment with rapamycin (AMPK inhibitor) increased the ratio of phospho-AMPK/total-AMPK. This result indicates that OGD/R activated phospho-AMPK and inhibits phospho-mTOR levels; inhibition of AMPK activated phospho-mTOR activity.

As shown in Figure 3(e) and Figure 3(f), the ULK1 was phosphorylated and inhibited at the Ser757 site by OGD/R and rapamycin. The expression of phospho-ULK1 were high in control group $(0.66 \pm 0.04)$, while the expression of phospho-ULK1 increased in model group $(0.11 \pm 0.02),(t=45.06, P<0.01)$ and rapa group $(0.20 \pm 0.01),(t=18.70, P<0.01)$; while inhibited AMPK $(0.30 \pm$ $0.04),(t=7.52, P<0.05)$ increased its levels. The results suggest that ULK1 were inhibited via interfering with the expression of AMPK and mTOR after OGD/R.

\subsection{Inhibition of AMPK Activity Reduced Cell Morphology Changes and Increases Cell Viability after OGD/R}

As shown in Figure 4(a), the cells grew very well, most of them were long fusiform, triangular or polygonal, with prominent cell protrusions and good adherence in
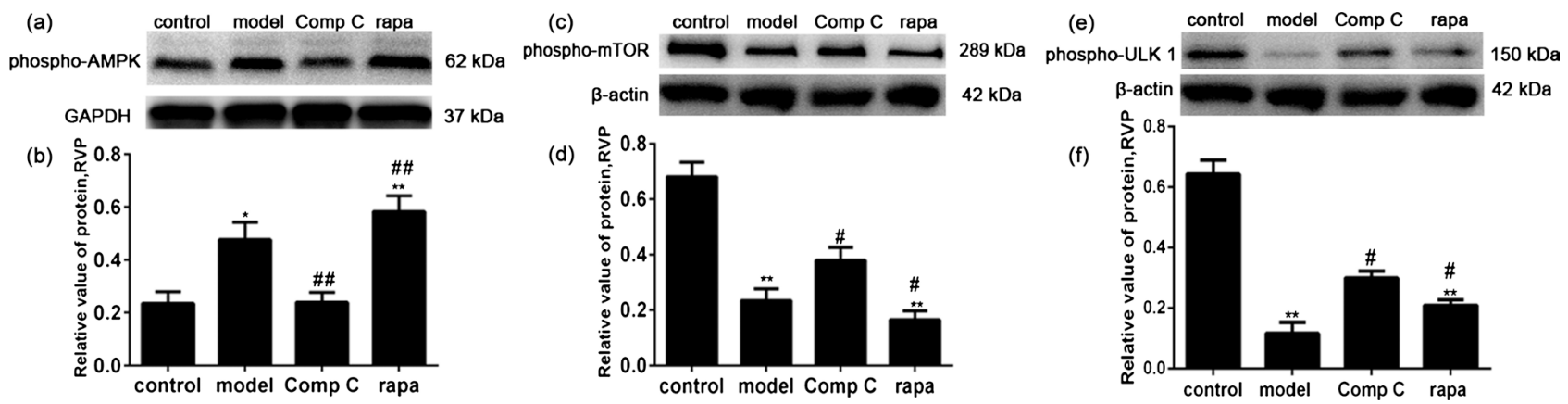

Figure 3. The AMPK-mTOR signaling pathway was involved in the process of OGD/R-induced autophagy. (a), (b): Representative immunoblots and quantification for analysis of phospho-AMPK, phospho-mTOR and phospho-ULK1 in SH-SY5Y cells after OGD/R. (c), (d): Representative immunoblots and quantification for analysis of phospho-mTOR in SH-SY5Y cells after OGD/R. (e), (f): Representative immunoblots and quantification for analysis of phospho-ULK1 in SH-SY5Y cells after OGD/R. Data are represented as mean \pm SEM from two independent experiments, ${ }^{\star} P<0.05$, ${ }^{\star *} P<0.01$ vs. control group; ${ }^{*} P<0.05$, ${ }^{\# \#} P<0.01$ vs. model group. Comp C: Compound C; rapa: rapamycin.
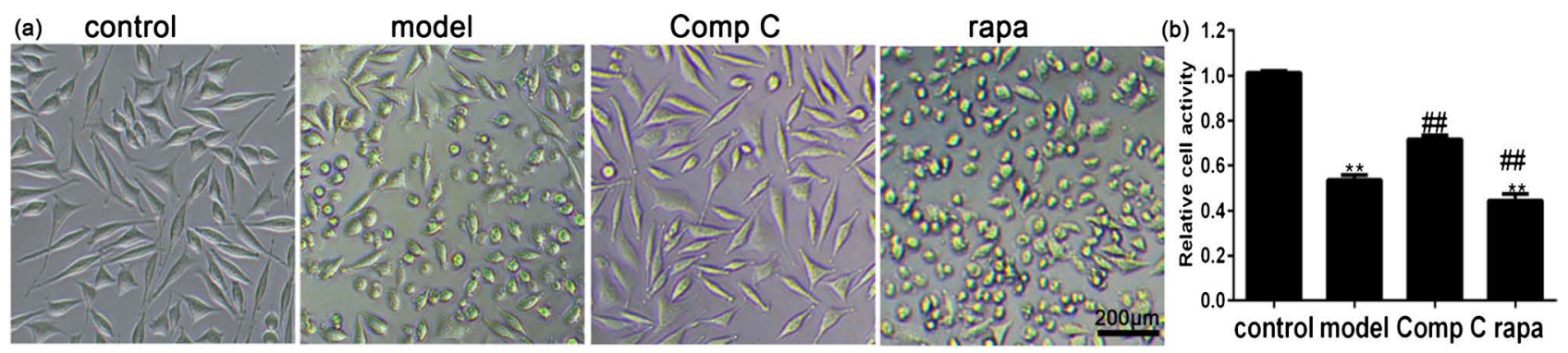

Figure 4. The effect of inhibition of AMPK activity on cells. (a): Morphological changes of cells after OGD/R. Scale bars: $200 \mu$ m; (b): Viability was determined by CCK-8 assay after OGD/R. Representative pictures and quantification for analysis are presented. Data are represented as mean \pm SEM from two independent experiments, ${ }^{\star} P<0.05,{ }^{\star *} P<0.01$ vs. control group; ${ }^{\#} P<0.05$, ${ }^{\# \#} P<$ 0.01 vs. model group. Comp C: Compound C; rapa: rapamycin. 
control group. In the model group, most of the cell gaps were enlarged, some cells were irregular in shape, wrinkled in volume, their protuberances were not obvious or even disappeared, and some cells were suspended from the wall in culture medium. Compared with the model group, after the Compound C pretreatment, the cell damage was significantly improved, only the cell gap became larger, the synaptic retraction and a small part of the cell deformation. Compared with the control group, the cell damage was significant, mainly as follows: most of the cell synapses disappeared, even atrophy to a round shape, and the number of exfoliated cells increased significantly.

It was shown in Figure 4(b) that the cell viability of the model group $(0.54 \pm$ $0.02)$ and rapa group $(0.44 \pm 0.03)$ were reduced, compared with the control group $(1.01 \pm 0.01)(t=36.11, P<0.01),(t=46.10, P<0.01)$. Pretreatment with Compound $\mathrm{C}(0.72 \pm 0.02)$ can increase cell viability, compared with the control group $(t=18.82, P<0.01)$.

\section{Discussion}

Autophagy is a way to obtain energy to maintain intracellular energy balance by degrading long-lived proteins and damaged organelles under stress conditions [18]. The occurrence of autophagy is related to autophagy-related gene (Atg). Autophagy flux, the dynamic process of autophagy, includes the formation of autophagosomes, the transport of autophagosomes to lysosomes, and the degradation of autophagic substrates in lysosomes. LC3, Beclin 1 are mainly autophagy markers. LC3, LC3, a homologue in Atg8 mammals, including both LC3I and LC3II. LC3 II binds to the autophagosome membrane intact and remains on the autophagosome until it is fused to lysosome. Beclin 1 is essential for the formation of autophagosomes [17]. Therefore, we detected autophagy by observing the expression of these autophagy-related proteins and the fluorescent dots of GFP-mRFP-LC3 adenovirus. Studies have shown that with the increase of autophagy-related proteins such as LC3 and Beclin 1, neuronal damage is aggravated in cerebral ischemia-reperfusion injury. Autophagy could enhance neuronal ischemic injuries, inhibiting autophagy flux alleviated neuronal injuries in Primary Cortical Neurons after OGD/R. These literatures suggest that up-regulated autophagy could aggravate cerebral ischemic injury. In our data, the Beclin 1, LC3 levels and GFP-mRFP fluorescent dots were increased, while abnormal cell morphology increased and cell viability decreased. The above results indicated that enhanced autophagy causes aggravation of nerve damage after OGD/R.

Accumulating evidence demonstrates that the AMPK-mTOR signaling pathway can regulate autophagy activation following cerebral ischemia-reperfusion injury [19] [20]. AMP can prevent the dephosphorylation of Thr172 in the AMPK $\alpha$ subunit and activate AMPK under stress such as ischemia and hypoxia [21]. Activated AMPK abolishes mTOR inhibition of autophagy [22]. Studies have shown that in rat cerebral ischemia-reperfusion injury, phospho-ULK1 was 
involved in the induction of autophagy, and its activity was regulated by mTOR [23]. The ULK1 complex includes ULK1, Atg13 and FIP200. When mTOR was inhibited, phosphorylation of ULK1 (Ser757) and Atg13 was blocked, ULK1 complex is activated, and autophagy is induced [24]. In our data, we found that phospho-AMPK was significantly activated after OGD/R, whereas the expression of phospho-mTOR was inhibited and the expression of phospho-ULK1 (Ser757) was significantly reduced. At the same time, the expression of autophagy-related proteins LC3 and Beclin 1 and the autophagic flux increased. Inhibition of AMPK activity activated mTOR, increased expression of phosphoULK1, decreased levels of autophagy related proteins and decreased autophagy flux. This indicates that the activation of autophagy by SH-SY5Y cell OGD/R via AMPK-mTOR signaling pathway may be related to the above mechanism.

Insufficient energy caused by ischemia and hypoxia is one of the important causes of nerve cell damage [25]. AMPK is a cellular energy sensor that changes the ratio of intracellular AMP/ATP. AMPK is activated by phosphorylation after cerebral ischemia in rats [11]. Activation of AMPK can acutely regulate cellular metabolism and long-term regulation of gene expression to restore ATP levels. AMPK enhances catabolism, inhibit anabolism, and maintain cell homeostasis [12]. In addition, it can induce autophagy, degrade autophagosomes to produce energy, and reduce dependence on external nutrition [12]. The energy crisis during cerebral ischemia induces autophagy, but ATP levels can be widely restored during reperfusion [26]. The recovery of ATP level can alleviate the energy crisis and reduce the brain damage caused by ischemia and hypoxia. However, recent studies have shown that excessive activation of AMPK causes autophagic apoptosis of neurons after cerebral ischemia [27]. Enhanced autophagy leads to neuronal apoptosis and exacerbates cerebral ischemic injury. Therefore, inhibition of AMPK down-regulation of autophagy may reduce cerebral ischemia-reperfusion injury.

In this data, we further observed the effect of inhibition of AMPK activity on cell status and autophagy after OGD/R. Treatment with Compound C downregulated the autophagy-related proteins LC3, Beclin 1 and autophagy flux, while down-regulating the expression levels of phospho-mTOR and ULK 1. In addition, abnormal cell morphology changes were reduced and cell viability was increased. mTOR promotes differentiation of neural stem cells and neuronal repair through epidermal growth factor(EGF) and fibroblast growth factor 2 (FGF2) [28]. The above results indicate that inhibition of autophagy can reduce nerve damage by down-regulating AMPK and up-regulating mTOR in SH-SY5Y cells after OGD/R.

\section{Conclusion}

In conclusion, inhibition of autophagy protects nerve cells from OGD/R damage in SH-SY5Y cells. This protection may be related to inhibition of AMPK activity by activation of mTOR and inhibition of autophagy. 


\section{Acknowledgements}

Thanks to Tingting Wang for providing language help and writing help.

\section{Funding}

This work was supported by the Clinical Medicine + X Project Fund of Qingdao Medical College (2017M016).

\section{Conflicts of Interest}

The authors declare that they have no conflict of interest to the work.

\section{Authors' Contribution}

"Yanxue Zhang" designed the study, performed the statistical analysis, wrote the protocol, and wrote the first draft of the manuscript. "Hongyun Li" performed for the revision of the experimental guidance and manuscript managed the analyses of the study. All authors read and approved the final manuscript.

\section{References}

[1] Feigin, V.L., Bo, N., George, M.G., Foltz, J.L., Roth, G.A. and Mensah, G.A. (2016) Prevention of Stroke: A Strategic Global Imperative. Nature Reviews Neurology, 12, 501-512. https://doi.org/10.1038/nrneurol.2016.107

[2] Kanazawa, M., Takahashi, T., Nishizawa, M. and Shimohata, T. (2014) Therapeutic Strategies to Attenuate Hemorrhagic Transformation after Tissue Plasminogen Activator Treatment for Acute Ischemic Stroke. Neurology \& Clinical Neuroscience, 1, 201-208.

[3] Yin, J., Tu, C., Zhao, J., Ou, D., Chen, G., Liu, Y., et al. (2013) Exogenous Hydrogen Sulfide Protects against Global Cerebral Ischemia/Reperfusion Injury via Its Anti-Oxidative, Anti-Inflammatory and Anti-Apoptotic Effects in Rats. Brain Research, 1491, 188-196. https://doi.org/10.1016/j.brainres.2012.10.046

[4] Chen, W., Sun, Y., Liu, K. and Sun, X. (2014) Autophagy: A Double-Edged Sword for Neuronal Survival after Cerebral Ischemia. Neural Regeneration Research, 9, 1210-1216.

[5] Pan, T., Kondo, S., Le, W. and Jankovic, J. (2008) The Role of Autophagy-Lysosome Pathway in Neurodegeneration Associated with Parkinson's Disease. Brain, 131, 1969-1978.

[6] Wen, Y.D., Sheng, R., Zhang, L.S., Han, R., Zhang, X., Zhang, X.D., et al. (2008) Neuronal Injury in Rat Model of Permanent Focal Cerebral Ischemia Is Associated with Activation of Autophagic and Lysosomal Pathways. Autophagy, 4, 762-769. https://doi.org/10.4161/auto.6412

[7] Petiot, A., Ogier-Denis, E., Blommaart, E.F., Meijer, A.J. and Codogno, P. (2000) Distinct Classes of Phosphatidylinositol 3'-Kinases Are involved in Signaling Pathways That Control Macroautophagy in HT-29 Cells. The Journal of Biological Chemistry, 275, 992-998.

[8] Jiang, W.W., Huang, B.S., Han, Y., Deng, L.H. and Wu, L.X. (2017) Sodium Hydrosulfide Attenuates Cerebral Ischemia/Reperfusion Injury by Suppressing Overactivated Autophagy in Rats. FEBS Open Bio, 7, 1686-1695.

[9] Li, H., Liu, X., Zhu, Y., Liu, Y. and Wang Y. (2015) Magnolol Derivative 002C-3 
Protects Brain against Ischemia-Reperfusion Injury via Inhibiting Apoptosis and Autophagy. Neuroscience Letters, 588, 178-183.

[10] Wang, M., Li, Y.J., Ding, Y., Zhang, H.N., Sun, T., Zhang, K., et al. (2016) Silibinin Prevents Autophagic Cell Death upon Oxidative Stress in Cortical Neurons and Cerebral Ischemia-Reperfusion Injury. Molecular Neurobiology, 53, 932-943.

[11] Fu, L., Huang, L., Cao, C., Yin, Q. and Liu, J. (2016) Inhibition of AMP-Activated Protein Kinase Alleviates Focal Cerebral Ischemia Injury in Mice: Interference with mTOR and Autophagy. Brain Research, 1650, 103-111. https://doi.org/10.1016/j.brainres.2016.08.035

[12] Wen, Y.A., Xing, X., Harris, J.W., Zaytseva, Y.Y., Mitov, M.I., Napier, D.L., et al. (2017) Adipocytes Activate Mitochondrial Fatty Acid Oxidation and Autophagy to Promote Tumor Growth in Colon Cancer. Cell Death \& Disease, 8, e2593. https://doi.org/10.1038/cddis.2017.21

[13] Wu, T., Wang, M.C., Jing, L., Liu, Z.Y., Guo, H., Liu, Y., et al. (2015) Autophagy Facilitates Lung Adenocarcinoma Resistance to Cisplatin Treatment by Activation of AMPK/mTOR Signaling Pathway. Drug Design Development \& Therapy, 9, 6421-6431. https://doi.org/10.2147/DDDT.S95606

[14] Sun, A., Li, C., Chen, R., Huang, Y., Chen, Q., Cui, X., et al. (2016) GSK-3 $\beta$ Controls Autophagy by Modulating LKB1-AMPK Pathway in Prostate Cancer Cells. Prostate, 76, 172-183. https://doi.org/10.1002/pros.23106

[15] Weiner, G.M. and Ducruet, A.F. (2015) Mammalian Target of Rapamycin (mTOR) Activity Promotes Neuronal Survival in Stroke with or without Ischemic Postconditioning. Neurosurgery, 76, N19-N20.

[16] Tasca, C.I., Dal-Cim, T. and Cimarosti, H. (2015) In Vitro Oxygen-Glucose Deprivation to Study Ischemic Cell Death. Methods in Molecular Biology, 1254, 197-210.

[17] Klionsky, D.J., Abdalla, F.C., Abeliovich, H., Abraham, R.T., Acevedoarozena, A., Adeli, K., et al. (2012) Guidelines for the Use and Interpretation of Assays for Monitoring Autophagy. Autophagy, 8, 445-544. https://doi.org/10.4161/auto.19496

[18] Dong, F., Yao, R., Yu, H. and Liu, Y. (2017) Neuroprotection of Ro25-6981 against Ischemia/Reperfusion-Induced Brain Injury via Inhibition of Autophagy. Cellular and Molecular Neurobiology, 37, 743-752.

[19] Liang, J., Shao, S.H., Xu, Z.X., Hennessy, B., Ding, Z., Larrea, M., et al. (2007) The Energy Sensing LKB1-AMPK Pathway Regulates p27(kip1) Phosphorylation Mediating the Decision to Enter Autophagy or Apoptosis. Nature Cell Biology, 9, 218-224.

[20] Hwang, J.Y., Gertner, M., Pontarelli, F., Court-Vazquez, B., Bennett, M.V., Ofengeim, D., et al. (2017) Global Ischemia Induces Lysosomal-Mediated Degradation of mTOR and Activation of Autophagy in Hippocampal Neurons Destined to Die. Cell Death \& Differentiation, 24, 317-329.

[21] Xiao, B., Heath, R., Saiu, P., Leiper, F.C., Leone, P., Jing, C., et al. (2007) Structural Basis for AMP Binding to Mammalian AMP-Activated Protein Kinase. Nature, 449, 496-500. https://doi.org/10.1038/nature06161

[22] Gwinn, D.M., Shackelford, D.B., Egan, D.F., Mihaylova, M.M., Mery, A., Vasquez, D.S., et al. (2008) AMPK Phosphorylation of Raptor Mediates a Metabolic Checkpoint. Molecular Cell, 30, 214-226.

[23] Yu, J., Bao, C., Dong, Y. and Liu, X. (2015) Activation of Autophagy in Rat Brain Cells Following Focal Cerebral Ischemia Reperfusion through Enhanced Expression of Atg1/pULK and LC3. Molecular Medicine Reports, 12, 3339-3344.

https://doi.org/10.3892/mmr.2015.3850 
[24] Russell, R.C., Yuan, H.X. and Guan, K.L. (2014) Autophagy Regulation by Nutrient Signaling. Cell Research, 24, 42-57.

[25] Doyle, K.P., Simon, R.P. and Stenzel-Poore, M.P. (2008) Mechanisms of Ischemic Brain Damage. Neuropharmacology, 55, 310-318.

[26] Zhang, X., Yan, H., Yuan, Y., Gao, J., Shen, Z., Shen, Y., et al. (2013) Cerebral Ischemia-Reperfusion-Induced Autophagy Protects against Neuronal Injury by Mitochondrial Clearance. Autophagy, 9, 1321-1333. https://doi.org/10.4161/auto.25132

[27] Manwani, B. and McCullough, L.D. (2013) Function of the Master Energy Regulator Adenosine Monophosphate-Activated Protein Kinase in Stroke. Journal of Neuroscience Research, 91, 1018-1029.

[28] Magri, L. and Galli, R. (2013) mTOR Signaling in Neural Stem Cells: From Basic Biology to Disease. Cellular \& Molecular Life Sciences, 70, 2887-2898.

https://doi.org/10.1007/s00018-012-1196-x 